\title{
Peak Nasal Inspiratory Flow
}

National Cancer Institute

\section{Source}

National Cancer Institute. Peak Nasal Inspiratory Flow. NCI Thesaurus. Code C119547.

The maximal flow achieved during the maximally forced inspiration through the nose initiated at maximum exhalation. 